\title{
Mesorectal hematoma associated with colic and caudal neurological signs in a horse
}

\author{
Max Diekstall' and Astrid Rijkenhuizen² \\ 1 Pferdeklinik Leichlingen, Leichlingen, Germany \\ 2 Wijk Bij Duurstede, The Netherlands
}

\begin{abstract}
Summary: A 6-year- old Warmblood gelding was referred for acute colic signs and stranguria. Clinical examination revealed reduced anal and tail tone as well as an intra-abdominal mass palpable per-rectum. The mass prevented the palpation of normal abdominal structures. The horse was subjected to standing diagnostic laparoscopy which revealed a retroperitoneal hematoma extending from the root of the mesentery to the mesorectum, bulging to the left side of the abdomen. The horse was initially managed medically, with the horse placed in box confinement under close monitoring. The colic signs subsided, however, a progressive paralysis of the horse's tail and the inability to defecate became worse. The horse subsequently underwent a diagnostic laparotomy where the origin of the hematoma could be localized in the mesorectum. The horse was euthanized intraoperatively due to the size and location of the hematoma. The worsening of the neurological signs was theorized to have been caused by compression of the neural structures by the expanding hematoma.
\end{abstract}

Keywords: Colic, retroperitoneal hematoma, laparoscopy, neural damage, intraabdominal mass, mesorectal hematoma

Citation: Diekstall M., Rijkenhuizen A. (2018) Mesorectal hematoma associated with colic and caudal neurological signs in a horse. Pferdeheilkunde 34, 232-236; DOI 10.21836/PEM20180303

Correspondence: Dr. Max Diekstall, Pferdeklinik Leichlingen, Am Further Weiher 1, 42799 Leichlingen, Germany; m.diekstall@pferdeklinik-leichlingen.de

\section{Introduction}

Abdominal masses like neoplasia, abscesses, or mesenteric hematoma are diagnosed during examination in horses with colic signs either through transrectal palpation or ultrasonography of the abdomen (Mueller 2002). Clinical signs associated with masses are related to the location of them and can initially be non-specific. Abdominal discomfort can be caused by tension on the mesentery or mechanical obstruction of the intestinal lumen (Garcia-Seco 2005). Diagnostic laparoscopy in the standing horse can be helpful to localize the cause of signs to initiate a treatment (Brehm 2017). This report describes the presence of a unique abdominal mass identified as a retroperitoneal hematoma in the mesorectum causing colic signs and neurological signs by compression of the caudal nerves. As to the authors' knowledge, these clinical findings have not previously been reported in equine literature. This case report describes the clinical signs and outcome.

\section{Case history}

A 6- year- old Warmblood gelding was referred for acute colic signs. The horse had been in the owner's possession since foal and did not have any prior abdominal problems. Teeth were checked every half year by a veterinarian and the horse was dewormed regularly. Castration had occurred as a 2-year-old without complications in the postoperative period. On the morning of referral the horse showed severe colic signs, like: lying down frequently, sweating and pawing at the ground with the forelimb. The horse was stretching out the abdomen, which was interpreted as stranguria by the referring veterinarian who had suspected a full bladder after perrectal examination and treated the horse with Scopolamin
Butylbromid (Buscopan compositum $5 \mathrm{ml} / 100 \mathrm{~kg}$ ) a and Metamizol (Novaminsulfon $5 \mathrm{ml} / 100 \mathrm{~kg}$ ) ${ }^{\mathrm{b}}$ intravenously. As the horse showed no improvement after 2 hours and colic signs remained, it was referred to the clinic. Whether the horse had subsequently urinated was unknown at the time of presentation.

\section{Clinical findings}

Upon presentation at the referral hospital the horse was sweaty and exhibited abdominal pain characterized by pawing at the ground with the forelimb and reaching around with the head to the flank. The horse had a normal body condition score of 375 (Anon 2005). A heart rate of 40 beats/ min, respiratory rate of 20 breaths/min and a rectal temperature of $37.8^{\circ} \mathrm{C}$ were noted, heart and lung auscultation was within normal limits. Oral mucus membrane were pink and the capillary refill time was less than 2 seconds. The packed cell volume (PVC) was $0,26 \mathrm{I} / \mathrm{I}$ (reference range (rr) $0,32-0,45 \mathrm{I} / \mathrm{I})$, total plasma protein concentration and lactate were $60 \mathrm{~g} / \mathrm{l}(\mathrm{rr} 52-68 \mathrm{~g} / \mathrm{l})$ and $2 \mathrm{mmol} / \mathrm{l}(\mathrm{rr}$ $0.5-1.78 \mathrm{mmol} / \mathrm{I})$ respectively.

Abdominal auscultation on the right and left flanks revealed intermittent peristaltic sounds. A reduced anal tone and tail flaccidity was noted when taking the rectal temperature. The horse showed no signs of ataxia or desensitization of other regions of the skin. The ampulla recti had no feces inside. Rectal mucus membranes were thickened through edematous swelling with a dry surface. It was difficult to examine the abdominal cavity because a mass from the dorsal peritoneal surface to the pelvis prevented further palpation of the abdomen cranially. The mass felt soft, dense and crushable and could not be balloted or pushed cranially. It was bulging to 
the left side of the abdomen. The bladder could not be palpated. Transabdominal ultrasonography from the ventral abdomen showed good motility of the bowel and an increased amount of fluid. Abdominal fluid was retrieved by abdominocentesis. The fluid was odorless and had a pale yellow color. Peritoneal fluid analysis yielded total nucleated cell count of $6000 / \mu \mathrm{L}$ (rr 5000 to 10000 cells $/ \mu \mathrm{L}$ ), total protein concentration of $24 \mathrm{~g} / \mathrm{L}(\mathrm{rr}<25 \mathrm{~g} / \mathrm{L})$ and lactate within normal limits $(1.5 \mathrm{mmol} / \mathrm{l}, \mathrm{rr}$ 0,65-1.7 mmol/I). Transrectal ultrasonography revealed a mass with a anechogenic content and a well demarcated capsule. The origin of the mass and its proximity to other structures could not be identified either by ultrasonography or by palpation.

Only small amounts of fluid $(300 \mathrm{ml})$ could be retrieved from the stomach with the nasogastric tube; the $\mathrm{pH}$ from the gastric fluid was 4 . The horse was taken to stable for observation. After 30 minutes the horse showed further signs of colic and on basis of the persistent abdominal pain and the transrectal findings a standing exploratory laparoscopy was elected. A laparotomy was considered less suitable to visualize the abdominal mass based on the pre-surgical findings of a dorsally positioned mass.

\section{Surgery}

Phenylbutazone (Phenylbutazon $200 \mathrm{mg} / \mathrm{ml} 4.4 \mathrm{mg} / \mathrm{kg}$ intravenously) ${ }^{c}$ and procaine penicillin $G$ (Procain Penicillin $22.000 \mathrm{U} / \mathrm{kg}$ intramuscularly) ${ }^{d}$ were administered prior to laparoscopy. The horse was sedated with detomidine hydrochloride (Detogesic $10 \mathrm{mg} / \mathrm{ml} 0.012 \mathrm{mg} / \mathrm{kg} \mathrm{IV}$ ) e and butorphanol tartrate (Torbugesic Vet $0.025 \mathrm{mg} / \mathrm{kg} \mathrm{IV}$ ) through a jugular vein catheter. Given that the mass seemed to extend into the left caudal abdomen, a left sided approach was chosen. The left flank was clipped and the gelding restrained in stocks with the tail tied up. After routine preparation for surgery with chlorhexidine and alcohol, the skin and underlying structures were infiltrated with $10 \mathrm{ml}$ of $2 \%$ lidocaine (Lidocainhydrochlorid $2 \%)^{g}$. The portal for the laparoscope was made half way between the last rib and the tuber coxae in the paralumbar fossa, just dorsal to the internal oblique abdominal muscle. A $2 \mathrm{~cm}$ skin incision was made and with a Metzenbaum scissor the muscles were bluntly divided in a caudoventral direction. The cannula with trocar $\left(10 \mathrm{~mm}\right.$ Dr. Fritz $\left.{ }^{\odot}\right)$ was inserted and its location within the abdomen was confirmed by detecting negative pressure and hearing the sound of air entering the abdomen. The trocar was replaced by the lapa-

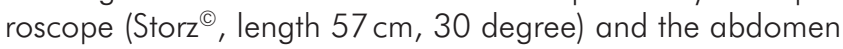
was insufflated with $\mathrm{CO}_{2}(1.6 \mathrm{~L} / \mathrm{min}$ pressure $)$ to a maximum pressure of $6 \mathrm{~mm} \mathrm{Hg}$.

Laparoscopic inspection of the left dorsal abdominal cavity revealed a retroperitoneal abdominal mass, extending dorsally from the mesenteric root (Fig. 1) to the pelvic inlet. The mass consisted of red shiny fluid contained within the retroperitoneal space. The size of the mass was at least two watermelons and depressed the large colon to the left side of the abdomen. Caudally the mesorectum was filled with the same fluid and was distended to the left mesenteric root (Fig. 2). The bladder could not be visualised. The abdominal fluid had a pale yellow color. The increased amount of fluid seen by ultrasound could not be appreciated in surgery. No other abnormalities could be found on the left side, however, due to the size of the mass it was not possible to move the laparoscope to the right side of the horse without risking rupture the mass. The visceral peritoneum that covered the fluid was under extreme tension and the surgeon decided against a puncture of the mass to avoid a rupture and risking loss of passive tamponade of the suspected hematoma. Since no further surgical measures seemed possible, conservative management was pursued. The abdomen was desufflated, the laparoscope and instruments removed and the skin incisions of the portal was closed using 2 simple interrupted sutures (Biosyn 3.5 metric).

\section{Postoperative Care}

The horse was stall rested and procaine penicillin G ( Procain Penicillin R 22.000 U/kg intramuscularly) was continued once daily for 3 days and Flunixin meglumine (Flunidol R RPS $50 \mathrm{mg} / \mathrm{ml} 1.1 \mathrm{mg} / \mathrm{kg} \mathrm{BW})^{\mathrm{h}}$ once per day for 9 days. Food was withheld for 12 hours, then the horse was fed with mash and small amounts of wet hay $(4 \times 1.5 \mathrm{~kg} /$ day $)$ and had free access to water all the time. No further signs of colic were noted and the horse defecated regularly and had no problems urinating.

The day after surgery a cystoscopy was performed as traumatic rupture of the ureters and adjacent vessels was considered

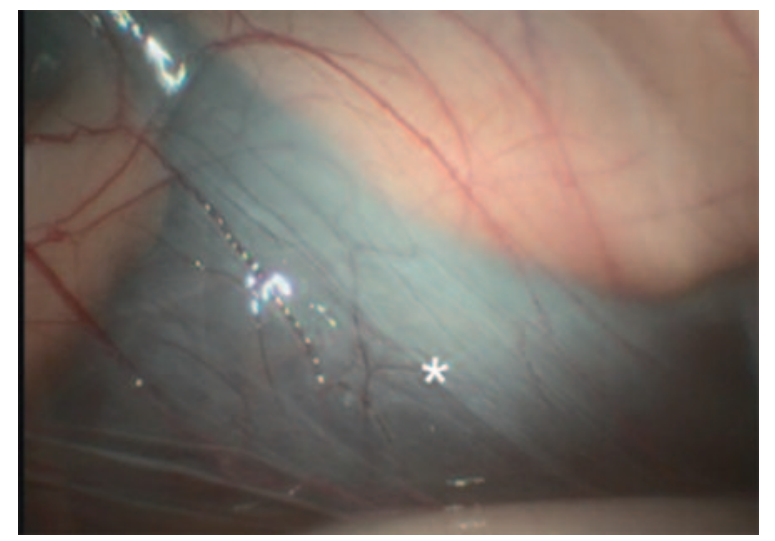

Fig. 1 Laparoscopic view of the cranial part of the left abdominal cavity showing the mesocolon $\left({ }^{*}\right)$ filled with red shiny fluid

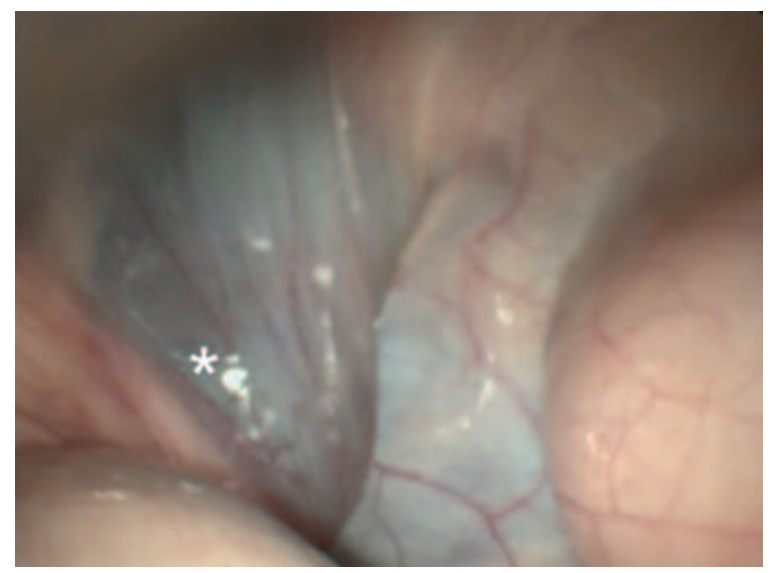

Fig. 2 Laparoscopic view of the caudal part of the left abdominal cavity showing the mesorectum $\left(^{*}\right)$ bulging laterally and filled with reddish fluid. 
as a cause for the retroperitoneal fluid. The dorsal wall of the bladder was collapsed and it was not possible to raise the dorsal wall through insufflation of the bladder with air through the endoscope. The mucosa of the bladder was shiny and both ureters appeared patent. Color of the urine was normal.

The first 3 days post-operatively the horse showed a dull demeanor, and required increased time for feed consumption. Rectal temperature stayed within normal limits $\left(37.5-38.2^{\circ} \mathrm{C}\right)$. The anal and tail tone remained reduced but the horse was able to lift the tail so far to defecate and urinate.

At day 3 colic signs recurred with a heart rate of 60 beats/min, sweating and lying down. Auscultation of the abdomen revealed decreased intestinal sounds. No gastric reflux could be obtained on passage of a nasogastric tube. The clinical signs of colic were eliminated using a single intravenous injection of Flunixin meglumine (Flunidol RPS $50 \mathrm{mg} / \mathrm{ml} \mathrm{R} 1.1 \mathrm{mg} / \mathrm{kg} \mathrm{BW).}$ Transrectal examination after Flunixin meglumine was limited still because of an unchanged extension of the mass. The ampulla recti was full with dry feces.

Hematological analysis was performed daily and was within normal limits or revealed no changes, except for the PVC. An initial decrease in PCV was observed the first post-operative day with $0,26 \mathrm{I} / \mathrm{I}$. After 5 days the PCV reached $0,34 \mathrm{I} / \mathrm{I}$ and stayed within normal limits.

The anal and tail tone decreased progressively daily from day 3 postoperative and at day 5 the horse had no tail tone and the horse began to be unable to defecate. To facilitate the passage of feces, $1 \mathrm{~L}$ of paraffin oil was introduced rectally using a nasogastric tube twice daily.

After 8 days the horse exhibited itching at the base of the tail either due to the inability to lift it while passing feces or to the manifestation of the neurological signs. The perineum was cleaned several times daily. At this time also colic signs reoccurred twice daily and fecal retention was considered to be the cause as the ability of passing the sphincter was reduced. It was then decided to empty the rectum manually every 8 hours.

Gradually the horse showed a progressive worsening of its clinical behavior, seemed to be dull demeanor and refused the food. Transabdominal ultrasonography of the mass at day 8 postoperative revealed no changes. As the horse lost its appetite and stopped to finish hay and mash completely, the intermittent signs of colic and the progressive neurological signs led to the decision to perform an exploratory laparotomy in order to define the abdominal state of the retroperitoneal hemorrhage and make a final decision.

The laparotomy was performed in dorsal recumbency. The hair was clipped and the ventral abdomen wall prepared aseptically for surgery. A midline approach extending from the umbilicus toward cranial was made and the abdominal cavity was explored. The stomach, cecum, ileum and jejunum were without any anomalies. After exteriorizing the ascending and descending colons, the mesocolon could be followed as far as the pelvic cavity. A fluctuating mass could be palpated in the mesorectum from the entrance to the pelvic cavity to the anus retroperitoneally, which was bulging over the rectum to the left side. With a $20 \mathrm{ml}$ syringe, an $18 \mathrm{G}$ needle and an extension tube (Heidelberger Verlängerung) the mass was punctured and $6 \mathrm{ml}$ of old blood was aspirated. The rest of the mass was built up of clotted blood. The diagnosis of a retroperitoneal hematoma was confirmed. Due to the size and the location of the hematoma, the inability to remove it in-toto and the progressive neurological signs it was decided, in conjunction with the owner, to euthanize the horse intraoperatively.

The owner allowed only a minimal post- mortem examination performed by the surgeon. The abdominal incision was enlarged cranially to the xiphoid cartilage and caudally to the symphysis of the pelvis. The pelvic symphysis was split to visualize the caudal aspect of the abdominal cavity. The descending colon was transected and closed using a ligature. The cranial bowel was completely removed. The retroperitoneal space of the rectum was opened and contained dark fluid with clots. On basis of the intraoperative findings and post- mortem examination diagnosis of a retroperitoneal hematoma was made.

\section{Discussion}

The retroperitoneal hematoma located in the mesorectum and mesocolon in the presented case was associated with severe colic signs and progressive neurological signs as reduced anal and tail tone. Laparoscopy showed a huge mass extending from the pelvis cranial to the mesenteric root and caudally the mesorectum was under extreme tension. Traction of the mesentery is known to induce strong autonomic reactions in human patients (Holzer-Petsche and Brodacz 1999). Additionally tension on the mesentery is known to cause pain and colic signs in horses (Mair 2002), especially when tension develops rapidly. The horse had severe colic signs at the time of referring, which means hemorrhage must have developed promptly, whereas slowly developing tension may be painless (Mair 2002). In human medicine there is an emergency case called abdominal compartment syndrome. The abdominal compartment syndrome is characterized by an accumulation of fluid either intraperitoneal or retroperitoneal (Tomoharu et al 2017). Symptoms are organ dysfunction, such as oligura, decreased oxygenation and progression of metabolic acidosis. For treatment of the abdominal compartment syndrome, improvement of the abdominal wall compliance through evacuation of the bowel and peritoneal contents, fluid management, improvement of organ perfusion and surgical decompression is recommended in human medicine. In our case the mass appeared to compress the bladder and urethras and cause stranguria. The endoscopic examination of the bladder the day postoperative confirmed this by the presence of collapse of the dorsal wall. At that time yellow, clear urine came out of both ureteral orifices and no impairment of urine flow was noted. During the following days the mass got smaller through organization of the hematoma and the signs of stranguria disappeared. However, it seems that even large amounts of retroperitoneal fluid in horses do not cause these acute syndromes like you can see in humans. In our case the tension of the mesentery seemed to cause the pain, the fluid was responsible for a progressive worsening of the neurological signs. 
Based on the findings of the laparotomy examination the hematoma was mainly localized in the mesorectum, consisting of loose, connective tissue (McKay et al 2003) with suspected increased pressure on the underlying nerves. The cause of the hemorrhage was uncertain but it was hypothesized that it had its origin from a mesenterial vessel running in the mesorectum and, or, mesocolon.

Retroperitoneal hemorrhage in the mesorectum and mesocoIon has been described to occur in humans and as far as the authors are aware this is the first report in equine literature. In humans hematoma and retroperitoneal hemorrhage are associated with cardiac and peripheral interventions like cardiac and peripheral vascular catheterization, trauma, underlying pathologies such as abdominal aortic aneurysm or coagulopathy. Hematoma is the most common complication and retroperitoneal hemorrhage has the highest mortality (Freilich et al 2012). The retroperitoneum can contain large amounts of blood before specific signs or signs occur (Freilich et al 2002). Therefore the diagnosis is often made late with fatal outcomes (Freilich et al 2002). In our case the cause of the hemorrhage could not be determined as no trauma or accident was reported. Given that, horses are not watched $24 \mathrm{~h}$ per day, trauma in the box or in the paddock could not be completely excluded.

The hematoma was most extensive caudal, where it bulge along the rectum and from the transrectal examination also pararectally. It was hypothesized that the initial trauma and resulting hematoma were caudal and as retroperitoneum is loose connective tissue it spread out cranially. As another cause for the hematoma a neoplasia of the vessels could be possible but due to the missing necropsy could not be proved. The results of the hematological examination support blood loss with a PCV of $0,26 \mathrm{l} / \mathrm{I}$ at the day of referral, which improved within 3 days.

The bleeding was self- limiting due to the tamponade effect of the retroperitoneum and over the days the horse spent in hospital absorption of the blood took place. From the second surgery on it became clear, that the hematoma had subsided cranially in the mesocolon and remained at the place of its origin. The conservative management as treatment seemed to have had a realistic chance, as the patient was initially hemodynamically stable. On the other hand it should be kept in mind that a spontaneous rupture of the mesocolon and drainage of the hematoma into the peritoneal cavity was a possibility. Additionally the hematoma, and its pararectal location was potential nidus of bacterial infection with the subsequent risk of abscess and septic peritonitis.

In the peritoneal part of the abdominal cavity the rectum is attached to the mesorectum. The rectum gets its arterial blood supply by the cranial rectal artery that comes from the caudal mesenteric artery. This passes over the mesocolon of the descending colon into the mesorectum (Nickel 2004). The venous blood flows, like all blood of the bowel, into the Vena portae and as a characteristic of the rectum also into the caudal vena cava. The transrectal palpation, the laparoscopy, the laparotomy and the pathological examination localized the hematoma to the mesorectum and the caudal mesocolon of the descending colon. It seemed obvious that one of these vessels had ruptured and that blood had tracked between the peritoneal layers forming the mesenterium. From human literature it is known that a failure to localize the site of the bleeding can result in death (Freilich et al 2002).

Unfortunately, in this case progressive neurological changes occurred, likely due to the concurrent pressure of the hematoma on the caudal nerves with the peritoneal cavity.

Initially the horse had a reduced anal reflex and a reduced tail tone but was still able to defecate and urinate. However the reflexes reduced until eventually there was no ability to defecate. Progressive neural damage was considered. The caudal rectal nerves are the most caudal branches of the sacral plexus. Especially in the horse and the pig the caudal rectal nerves deliver the Ramus musculi coccygei and the Ramus musculi levatoris ani (Nickel 2004). In the dog these nerves are independent branches of the first sacral nerve. The ramus musculi coccygei is responsible for the medial and lateral coccygeus muscle and for the skin around the anus. The Ramus musculi levatoris ani innervates the $M$. levator ani and supplies the caudal part of the rectum. Furthermore the $\mathrm{N}$. pudendus deliver the $\mathrm{Nn}$. perineales superficialis that is responsible for muscles in the perianal region. At the first examination of the horse just the tail tone was reduced, over the time the horse stayed in hospital it developed that the horse was disable to lift its tail at all. This suggests that these nerves were not directly damaged by an acute trauma but were compressed by surrounding edema and/or hematoma resulting in a neuropraxia or axonotmesis. The horse showed no signs of ataxia or desensiveness of other regions of the skin and the neurological problems could be located to the $\mathrm{Nn}$. rectales caudales and the N. pudendus. It was hypothesized that the horse could have had a chance to survive if there were no progressive neurological problems, causing the disabiltity to lift the tail at all.

Further diagnostic modalities such as myelography were considered unnecessary given the progressive neurological signs and an inability to lift the tail.

Differential diagnostic a hemangiosarcoma could be kept in mind, which is also known to be a problem in horses (Southwood et al 2000) and are known to be initially be treated as hematomas and don ' $\dagger$ necessarily present as discrete tumors. Cytological examination of the fluid aspirated from the mass would have been an option to confirm the presence of tumor cells. Theoretically a rupture of the ureters could have caused a retroperitoneal swelling as well, however, at the time of examination, the ureters were intact and the horse urinated normally.

\section{Acknowledgements}

The authors would like to thank James Carmalt from the University of Saskatchewan for correcting the English language.

\section{Manufacturers`addresses}

a Buscopan ${ }^{\circledR}$ compositum: Boeringer Ingelheim, HermannEhlers- Straße 5, 41749 Viersen, Germany

b Novaminsulfon ${ }^{\circledR}$ : bela-pharm GmbH \& Co.Kg, Lohner StraBe 19, 49377 Vechta, Germany 
c Phenylbutazon ${ }^{\circledR} 200 \mathrm{mg} / \mathrm{ml}$ : medistar, Lüdinghauser Straße 23, 59387 Ascheberg

d Procain-Penicillin- $\mathrm{G}^{\circledR} 300,0 \mathrm{mg} / \mathrm{ml}$ : bela-pharm $\mathrm{GmbH}$ \& Co.Kg, Lohner Straße 19, 49377 Vechta, Germany

e Detogesic ${ }^{\circledR} 10 \mathrm{mg} / \mathrm{ml}$ : Zoetis, Schellingstraße 1, 10785 Berlin, Germany

${ }^{f}$ Torbugesic ${ }^{\circledR}$ Vet: Zoetis, Schellingstraße 1, 10785 Berlin, Germany

9 Lidocainhydrochlorid 2\%: bela- pharm GmbH \& Co.Kg, Lohner Straße 19, 49377 Vechta, Germany

h Flunidol ${ }^{\circledR} P S 50 \mathrm{mg} / \mathrm{ml}$ : cp-pharma, Ostlandring 13, 31303 Burgdorf, Germany

\section{References}

De Blaauw J. A., Rijkenhuizen A. B., van Nieuwstadt R. A., Grinwis G. C., Back W. (2003) Malignant melanoma of the pelvis as a possible cause of pelvic limb lameness in two gray mares. Tijdschr. Diergeneeskd. 128, 512-518

Freilich M. I., Gossmann D. E., Applegate R. J., Werns S. W., Gips S. J., Turi Z. G. (2012) The "Bladder Sign "- An Important Early Marker of Retroperitoneal Hemorrhage. Catheteriz. Cardiovas. Interv. 79, 158-165; DOI 10.1002/ccd.23141
Garcia-Seco E., Wilson D. A., Kramer J., Keegan K. G., Branson K. R., Johnson P. J., Tyler J. W. (2005) Prevalence and risk factors associated with outcome of surgical removal of pedunculated lipomas in horses: 102 cases (1987-2002). J. Am. Med. Vet. Assoc. 226, 1529-1537

Becker, M. Scheidemann, W. Stadtbäumer G. (2017) Diagnostik des Kolikpferdes. In: Handbuch Pferdepraxis, 4. Auflage 2017. Eds: Brehm W., Gehlen H., Ohnesorge B., Wehrend A., Enke-Verlag: 492, 493

Holzer-Petsche U, Brodacz B. (1999) Traction on the mesentery as a model of visceral nociception. Pain 80, 319-328

McKay J. S., Clegg P. D., Morthole V. I., Blake C. L. (2003) Primary retroperitoneal tumor in a horse. Equine Vet. J. 35, 103-106; DOI $10.1111 /$ petr. 12180

Mueller E. (2002) Rectal examination. In: Manual of Equine Gastroenterology, Vol. 1, 1st edn., Eds: Mair T., Divers T., Ducharme N., WB Saunders: 6

Nickel R. (2004) Nerven der Hintergliedmaße. In: Lehrbuch der Anatomie der Haustiere, Band IV Nervensystem, Sinnesorgane, Endokrine Drüsen. Eds: Nickel, R., Schummer, A., Seiferle ,E., Parey: 200, 289

Southwood L. L., Schott II H. C., Henry C. J., Kennedy F. A., Hines M. T., Geor R. J., Hassel D. M. (2000) Disseminated Hemangiosarcoma in the Horse: 35 Cases. J. Vet. Intern. Med. 14, 105-109

Tomoharu S., Kiyoto B., Seiichi Y., Chie A. (2017) Abdominal compartment syndrome during hip arthroscopy for an acetabular fracture: a case report. JA Clin. Rep. 3, 24; DOI 10.1186/s40981017-0100-y 